\title{
Capacidad de Penetración y Difusión de la Medicación, Intraconducto en Túbulos Dentinales, Conductos Laterales e Istmos. Una Revisión Sistemática
}

\author{
Penetration and Diffusion Capacity of Intra-duct Medication in \\ Dentinal Tubules, Lateral Canals and Isthmus. A Systematic Review
}

Luis Felipe Jiménez Rojas ${ }^{1,2}$; María Del Pilar Juarez² \& Flavio Rodrigues Ferreira Alves ${ }^{2}$

JIMÉNEZ, R. L. F.; JUAREZ, M. P. \& FERREIRA, A. F. R. Capacidad de penetración y difusión de la medicación intraconducto en túbulos dentinales, conductos laterales e istmos. Una revisión sistemática. Int. J. Odontostomat., 15(3):727-733, 2021.

RESUMEN: El objetivo de la terapia endodóntica consiste en tratar o prevenir la periodontitis apical. La efectiva eliminación o disminución de microbiota es esencial para la reparación periapical. Debido a esto, se utiliza el empleo de medicamentos intraconductos para tratar químicamente el sistema de conductos radiculares, y cobra relevancia la capacidad de penetración y difusión de dicho medicamento para entrar en contacto directo con los microorganismos que penetren dentro de los túbulos dentinales, conductos laterales e itsmos. Se realizó una revisión de la literatura en bases de datos electrónicas con los buscadores "Pubmed, Scielo y Google Académico", utilizando palabras clave para identificar la literatura publicada relevante en inglés. El análisis y los criterios de elegibilidad se documentaron de acuerdo con los elementos de informe para revisiones sistemáticas y meta-análisis (Preferred Reporting Items for Systematic Reviews and MetaAnalysis, PRISMA). No se impusieron restricciones sobre la fecha de publicación. Se recogieron datos sobre capacidad de difusión de medicación intraconductos en túbulos dentinales, conductos laterales e itsmos radiculares y se sometieron a análisis descriptivo de datos. La búsqueda bibliográfica en bases de datos electrónicas según los criterios de inclusión arrojó 45 títulos y resúmenes. Entre ellos, 7 artículos fueron seleccionados. Se determinó un rango de penetración del medicamento muy variable, entre $27,7-2350 \mu \mathrm{m}$ y rango de difusión de $200-500 \mu \mathrm{m}$. Hay pocos estudios publicados que reporten la capacidad de penetración y difusión de la medicación intraconductos. El vehículo empleado y diámetro de las partículas en la preparación del medicamento y la eliminación de la capa de desecho aumentaría la capacidad de penetración/difusión del medicamento intraconductos dentro del túbulo dentinal, conductos laterales e itsmo.

PALABRAS CLAVE: conducto lateral, istmo radicular, medicación intraconductos, penetración, difusión, túbulo dentinal.

\section{INTRODUCCIÓN}

El objetivo de la terapia endodóntica consiste en tratar o prevenir la periodontitis apical (Lasala, 1971; Wu et al., 2006). Por supuesto, la efectiva eliminación o disminución de microbiota es esencial para la reparación periapical (Peters et al., 2000; Siqueira Jr. et al., 2001; Mohammadi \& Abbott, 2009). Todavía, el remanente infeccioso en las áreas apicales inaccesibles y complejidades anatómicas del conducto radicular, túbulos dentinarios, conductos laterales y accesorios, itsmos y deltas apicales pueden no ser completamente eliminado solo con la preparación quí- mica y mecánica (Wu et al.). Las bacterias ubicadas en estas áreas están protegidas de los efectos de las células y moléculas de defensa del huésped, los antibióticos administrados sistémicamente y de la acción de los instrumentos e irrigantes (Siqueira Jr. \& Lopes, 1999).

En este contexto, el empleo de medicamentos intraconductos se ha indicado como una estrategia entre sesiones para promover una reducción adicional de la carga bacteriana, antes de la obturación del

\footnotetext{
${ }^{1}$ Department of Endodontics, Universidad Central de Venezuela, Venezuela.

${ }^{2}$ Postgraduate Program in Dentistry, University of Grande Rio (UNIGRANRIO), Rio de Janeiro, RJ, Brazil.
} 
conducto (Sjögren et al., 1991; Siqueira Jr. \& Lopes; Estrela et al., 2001; Peciuliene et al. 2001). Además, algunos medicamentos intraconducto, como el hidróxido de calcio, disminuyen la inflamación periapical, reduciendo el dolor postoperatorio, y estimulan la sanación de los tejidos periapicales (Byström \& Sundqvist, 1981; Siqueira Jr. et al., 1997; Siqueira Jr. J. \& Lopes; Law \& Messer, 2004; El Karim et al., 2007; American Association of Endodontists, 2011).

Para que tengan eficacia, las medicaciones intraconducto deben difundir por todas las áreas anatómicas del sistema de conductos y también en los túbulos dentinarios (Siqueira Jr. \& Lopes). No existen estudios previos en la literatura que recopilen y discutan los resultados de los estudios que evaluaran la capacidad de penetración y difusión de medicaciones intraconducto. Por supuesto, el objetivo de la presente investigación fue revisar sistemáticamente la literatura sobre la capacidad de penetración y difusión de las medicaciones intraconducto en los túbulos dentinales, conductos laterales e istmos.

\section{MATERIAL Y MÉTODO}

Estrategia de Búsqueda. La búsqueda electrónica de la literatura fue realizada por los buscadores "PubMed, Scielo y Google Académico" investigando los siguientes términos: drug diffusion in endodontic, dental tubule medication, inter appointment medicaments, intra canal dressing, drug diffusion in isthmus and lateral canals, isthmus and lateral canals in endodontic, drug penetration in isthmus and lateral canals. Fueron considerados artículos publicados en inglés. Las búsquedas electrónicas se realizaron en noviembre y diciembre de 2020. No se impusieron restricciones a la fecha de publicación.

Se desarrolló un protocolo detallado para esta revisión sistemática en el que se establecieron y documentaron el análisis y los criterios de elegibilidad, de acuerdo con las pautas Preferred Reporting Items for Systematic Reviews and Meta-Analysis (PRISMA) y The Cochrane Collaboration (Liberati et al., 2009).

Los criterios PICOS se construyeron como se indica en el texto:

1.Participantes: conductos radiculares en raíces de dientes extraídos.

2. Intervención / exposición: exposición de las mues- tras a los diferentes medicamentos intraconductos, con actividad antimicrobiana en infecciones del conducto radicular.

3. Comparaciones: tratamiento con medicamentos de uso común, empleados solos o combinados, con vehículos inertes y/o activos.

4. Resultado: penetración de la medicación intraconductos en túbulos dentinales, conductos laterales e istmos, para su evaluación por medio de observación directa con diferentes métodos de microscopía y por cultivo de bacterias viables en placas de agar contando las unidades formadoras de colonias.

5. Diseño del estudio: todos los estudios experimentales realizados por procedimientos válidos.

Selección y Análisis de Datos. La investigación actual se centra en diferentes tipos de medicamentos intraconductos (pastas de hidróxido de calcio, clorhexidina, paramonoclorofenol alcanforado, chitosan, tetraciclina) que se utilizan con fines antimicrobianos en las infecciones del conducto radicular. Para refinar aún más la búsqueda, se adoptaron los siguientes criterios de inclusión: todos los artículos científicos que indiquen la capacidad de penetración/difusión de medicamentos intraconductos en túbulos dentinales, conductos laterales e istmos.

Para la búsqueda electrónica se emplearon las siguientes frases: dentinal tubule medication, drug diffusion in endodontic, inter-appointment medicaments, drug diffusion in isthmus and lateral canals, isthmus and lateral canals in endodontic, drug penetration in isthmus and lateral canals. Los datos recopilados fueron analizados descriptivamente por un examinador, de forma independiente.

Una vez obtenido los artículos científicos, se procedió a analizar el título y resúmenes de los mismos, para elegir los artículos que cumplieran los criterios de elegibilidad, a los cuales se les realizó lectura completa y se le aplicaron los siguientes criterios de exclusión: estudios que solo evalúen zona de inhibición, penetración/difusión de materiales endodónticos/odontológicos no relevantes, capacidad de penetración/difusión sin especificar medida cuantificable, medicación evaluada no actúa en contacto directo con las paredes internas del conducto radicular, estudios que evalúen penetración/difusión solo de vehículos y estudios de revisión bibliográfica. Los criterios de inclusión y exclusión y el flujo de estudios para la revisión sistemática se muestran en la Figura 1. 


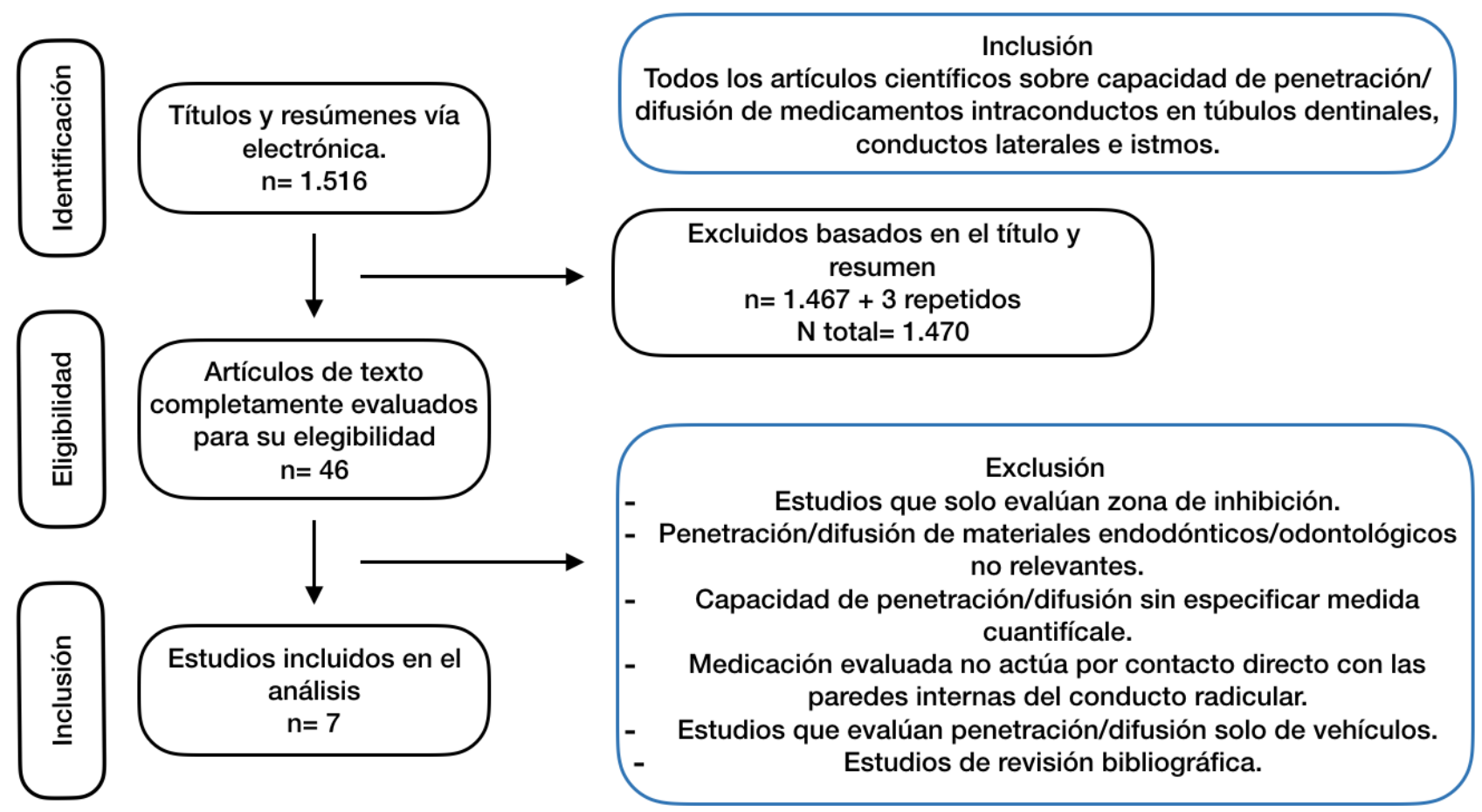

Fig. 1. Criterio de inclusión y exclusión y flujo de estudio para una revisión sistemática.

\section{RESULTADOS}

Identificación de Estudios. De los 1.516 resultados de la búsqueda electrónica, posterior a la lectura del título y sus resúmenes para determinar su relevancia, 46 artículos se consideraron relevantes para la investigación. Posterior a la completa lectura del artículo, se excluyeron 38 estudios por presentar criterios de exclusión descritos previamente. La búsqueda final electrónica y manual dio como resultado 7 artículos de investigación para su uso en este estudio.

Los criterios evaluados comprenden la capacidad de penetración o difusión de medicamentos intraconductos, solos o medicamentos combinados, que presenta rango de penetración expresado en micrómetros $(\mu \mathrm{m})$ y método de evaluación por medio de observación con microscopía en sus diferentes presentaciones y por medio de cultivo de bacterias viables en placas de agar contando las unidades formadoras de colonias.

De los 46 artículos relevantes para la presente investigación, 37 fueron publicados en revistas odontológicas, 7 publicados en revistas no odontológicas, y 2 corresponde a trabajo especial de grado publicado en la página web de una universidad; de los artículos publicados en revistas científicas, 18 fueron publicadas en revistas exclusivas del área endodóntica y 26 en revistas odontológicas no exclusivas del área endodóntica.

Con respecto a los 39 artículos excluidos, 13 fueron excluidos por ser estudios que solo evaluaron zona de inhibición del medicamento, 3 estudios que evaluaron difusión de materiales endodónticos/ odontológicos no relevantes, 13 estudios que no especificaron con medida cuantificable la capacidad de penetración/difusión del medicamento, 7 estudios cuya medicación evaluada no actúa en contacto directo con las paredes internas del conducto radicular, 1 estudio que evalúa la penetración/difusión solo del vehículo del medicamento y 2 estudios de revisión bibliográfica.

Penetración/Difusión. Se consideró la evaluación de las muestras por medio de observación con microscopía en sus diferentes presentaciones de microscopio electrónico de barrido (2 estudios), microscopio de escaneo de láser confocal (1 estudio), mi- 
croscopio de luz (1 estudio), microscopio de inmersión de aceite (1 estudio), cultivo de bacterias viables en placas de agar contando las unidades formadoras de colonias ( 2 estudio); de los cuales, 6 estudios fueron realizados con metodología In vitro (86\%) y un estudio con metodología in vivo (14\%).
El rango de penetración del medicamento presentó valores muy variables, entre $27,7-2350 \mu \mathrm{m}$ (Haapasalo \& Orstavik, 1987; Lin et al., 2003; Zand et al., 2017; Sungur et al., 2017; Sireesha et al., 2017; Arathi et al., 2019) (Tabla I), y el rango de difusión de 200-500 $\mu \mathrm{m}$ (Fuss et al., 2002) (Tabla II).

Tabla I. Penetración de la medicación intraconductos dentro del túbulo dentinal, conductos laterales e itsmo.

\begin{tabular}{|c|c|c|c|c|}
\hline Autor & Medicamento & $\begin{array}{l}\text { Rango de } \\
\text { penetración }\end{array}$ & Evaluación & $\begin{array}{l}\text { Tipo de } \\
\text { estudio }\end{array}$ \\
\hline Arathi et al. 2019 & $\begin{array}{l}\text { Clorhexidina al } 2 \% \\
\text { Chitosan }\end{array}$ & $\begin{array}{l}1800-2350 \mu \mathrm{m} \\
44,8-1250 \mu \mathrm{m}\end{array}$ & $\begin{array}{l}\text { Microscopio de inmersión } \\
\text { de aceite }\end{array}$ & In vitro \\
\hline $\begin{array}{l}\text { Zand et al. } \\
2017\end{array}$ & $\begin{array}{l}\text { Hidróxido de calcio + solución salina } \\
\text { Hidróxido de calcio nanopartículas + solución }\end{array}$ & $\begin{array}{l}27,7-64,1 \mu \mathrm{m} \\
79,3-284,5 \mu \mathrm{m}\end{array}$ & $\begin{array}{l}\text { Microscopio electrónico de } \\
\text { barrido }\end{array}$ & In vivo \\
\hline $\begin{array}{l}\text { Sungur et al. } \\
2017\end{array}$ & $\begin{array}{l}\text { Hidróxido de calcio + agua destilada } \\
\text { Hidróxido de calcio + propilenglicol }\end{array}$ & $\begin{array}{l}2059 \mu \mathrm{m} \\
1369 \mu \mathrm{m}\end{array}$ & $\begin{array}{l}\text { Microscopio de escaneo } \\
\text { de láser confocal }\end{array}$ & In vitro \\
\hline $\begin{array}{l}\text { Sireesha et al. } \\
2017\end{array}$ & $\begin{array}{l}\text { Hidróxido de calcio. } \\
\text { Hidróxido de calcio nanopartículas. } \\
\text { Chitosan } \\
\text { Chitosan nanopartículas }\end{array}$ & $\begin{array}{l}112-459 \mu \mathrm{m} \\
134-747 \mu \mathrm{m} \\
97-285 \mu \mathrm{m} \\
116-477 \mu \mathrm{m}\end{array}$ & $\begin{array}{l}\text { Microscopio electrónico de } \\
\text { barrido de emisión de } \\
\text { campo }\end{array}$ & In vitro \\
\hline Lin et al. 2003 & $\begin{array}{l}\text { Clindamicina } \\
\text { Tetraciclina }\end{array}$ & $\begin{array}{l}400 \mu \mathrm{m} \\
100-400 \mu \mathrm{m}\end{array}$ & $\begin{array}{l}\text { Cultivo de bacterias } \\
\text { viables en placas de agar y } \\
\text { contando las unidades } \\
\text { formadoras de colonias }\end{array}$ & In vitro \\
\hline $\begin{array}{l}\text { Haapasalo \& } \\
\text { Ørstavik } 1987\end{array}$ & $\begin{array}{l}\text { Paramonoclorofenol alcanforado líquido } \\
\text { Paramonoclorofenol alcanforado gaseoso } \\
\text { Hidróxido de calcio }\end{array}$ & $\begin{array}{l}+ \text { de } 700 \mu \mathrm{m} \\
400 \mu \mathrm{m} \\
100 \mu \mathrm{m}\end{array}$ & Microscopio de luz & In vitro \\
\hline
\end{tabular}

Tabla II. Difusión de la medicación intraconductos dentro del túbulo dentinal, conductos laterales e itsmo.

\begin{tabular}{|c|c|c|c|c|}
\hline Autor & Me dicamento & Rango de difusión & Evaluación & $\begin{array}{l}\text { Tipo de } \\
\text { estudio }\end{array}$ \\
\hline $\begin{array}{l}\text { Fuss et al., } \\
2002\end{array}$ & $\begin{array}{l}\text { Hidróxido de calcio + yodo yoduro de potasio } \\
\text { Hidróxido de calcio + cobre activado }\end{array}$ & $\begin{array}{l}200 \mu \mathrm{m} \\
500 \mu \mathrm{m}\end{array}$ & $\begin{array}{l}\text { Cultivo de bacterias } \\
\text { viables en placas de } \\
\text { agar y contando las } \\
\text { unidades formadoras } \\
\text { de colonias }\end{array}$ & in vitro \\
\hline
\end{tabular}

\section{DISCUSIÓN}

La medicación intraconducto consiste en la aplicación tópica de medicamentos dentro del sistema de conductos radiculares (SCR) con la intención de lograr efectos terapéuticos locales y no a nivel sistémico. Estos medicamentos pueden ser de diferentes tipos, tales como antisépticos, antibióticos, corticosteriodes y pastas de hidróxido de calcio para tratar el dolor, inflamación o para reducir la hemorragia. Los antisépticos constituyen la medicación tópica más empleada en endodoncia (Siqueira Jr. \& Lopes; Seltzer, 2004; American Asociation of Endodonticts).

Diversos estudios indican que el objetivo principal de la medicación intraconducto entre sesiones consiste en erradicar la mayor cantidad de bacterias y microorganismos, que probablemente con la preparación químico-mecánica, no se logran eliminar (Sjögren et al.; Siqueira Jr. \& Lopes; Estrela et al.; Peciuliene et al.).

El empleo local de sustancias irrigantes y quelantes son coadyuvantes químicos en el proceso de instrumentación del SCR (Siqueira Jr. \& Lopes; Seltzer). La utilización de hipoclorito de sodio, como irrigante durante la preparación química, puede penetrar entre $77 \mu \mathrm{m}$ hasta un máximo de $300 \mu \mathrm{m}$ dentro de los túbulos dentinales, dependiendo de la temperatura, tiempo y concentración (Zou et al., 2010). 
Para una desinfección completa de la dentina, los medicamentos aplicados localmente deben penetrar en la dentina en concentraciones suficientemente altas para eliminar las bacterias que invaden (Portenier et al., 2002)y la medicación debe estar en contacto directo con el microorganismo (Siqueira Jr. \& Lopes). Para conservar su actividad antibacteriana, el desinfectante debe resistir la inactivación por parte de la dentina y sus diversos componentes (Portenier et al.). Un medicamento que penetra más profundamente en los túbulos dentinales no solo puede servir como agente bloqueante, evitando la repoblación microbiana, sino que también los inactiva en los túbulos. Zand et al. indican que para lograr un efecto favorable, la medicación intraconductos de hidróxido de calcio debe difundir en los túbulos dentinarios para entrar en contacto directo con los microorganismos.

Por estas razones, es interesante determinar la capacidad de penetración y difusión de la medicación intraconductos dentro del túbulo dentinal, conductos laterales e itsmo para combatir a los microorganismos, que pueden penetrar en la longitud de los mismos, en un rango de 100-612 $\mu \mathrm{m}$ (Haapasalo \& Orstavik; Ørstavik \& Haapasalo, 1990; Sen et al., 1995; Siqueira Jr. et al., 1996; Al-Nazhan et al. 2014; Ran et al., 2015; Brittan et al. 2016), reproducirse y liberar toxinas que perpetúen la inflamación periapical (Ørstavik \& Haapasalo; Sen et al.; Peters et al.; Brittan et al.). Los microorganismos dentro de los túbulos dentinales, se encuentran protegidos de la acción de los instrumentos endodónticos y soluciones irrigadoras (Siqueira Jr. et al., 1996).

La habilidad del medicamento intraconductos de disolver y difundir en el SCR es esencial para que su acción sea exitosa (Siqueira Jr. \& Lopes; Abbott \& Yu, 2007), una vez que los microrganismos localizados en el interior de los conductos están protegidos de los mecanismos de defensa del hospedero o de los mismos agentes antimicrobiales de administración sistémica (Oguntebi, 1994; Fuss et al.).

Se destaca la necesidad de remover la capa de desecho antes de introducir el medicamento dentro del conducto radicular, si se desea lograr el efecto máximo (Abbott et al., 1989; Cruz et al., 2002). Puapichartdumrong et al. (2005), demostraron que la presencia de componentes pulpares remanentes tienen una influencia significativa sobre la difusión del medicamento en la superficie de la dentina (Puapichartdumrong et al.). Sin embargo, Oguntebi demostró que no hay una completa inhibición de la acción de los medicamentos intraconductos por la presencia de la capa de desecho en el conducto radicular, lo cual impediría la permeabilidad del túbulo dentinal; sin embargo, la remoción de esta capa de desecho ha demostrado ser importante para asegurar la desinfección del conducto. Biella et al. (2010), concluyen en su estudio in vitro que la utilización de radicación de laser de granate $(G)$ compuesto por Itrio (Y), Escandio (S) y Galio (G), contaminado con Erbio (Er) y Cromo (Cr) (Láser de Er,Cr:YSGG) remueve efectivamente detritos pulpares y la capa de desecho ubicados en el conducto radicular e incrementa la permeabilidad de la dentina, siendo recomendada su utilización para un uso clínico (Biella et al.).

Abbott et al. (1988) indicaron que la capacidad de difusión de los medicamentos no depende de la edad o sexo del individuo, sino del área de contacto directo entre el medicamento y la pared de dentina. A diferencia Wantulok et al. (1972), indican que los vapores de medicación intraconductos de metacresilacetato (Cresatin) y paramonoclorofenol alcanforado (CMCP), tienen la capacidad de difundir sus vapores a través del conducto radicular en su estudio in vitro (Wantulok et al.).

El vehículo empleado para mezclar junto al medicamento, el cual puede ser activo o inerte, es en parte, el encargado de la difusión de la medicación dentro del túbulo dentinal. de Souza Batista et al. (2014), demuestran que las pastas de solución salina y propilenglicol se difundían a través de los túbulos dentinarios. Como resultado de su estudio in vitro, las pastas de hidróxido de calcio experimentales que contienen aloe-vera pudieron permitir la difusión de iones hidroxilo a través de los túbulos dentinarios (de Souza Batista et al.; Brittan et al.).

Cruz et al. destacan en su estudio in vitro el área y la profundidad de penetración de un tinte con propilenglicol, el cual fue significativamente mayor que cuando se mezcló con agua destilada, concluyendo que el propilenglicol utilizado como vehículo a través del SCR es rápido y más eficaz, lo que indica su uso potencial para administrar medicamentos intraconductos.

A diferencia, Sungur et al. concluyen que tanto el propilenglicol como el agua destilada podrían usarse como vehículo y mostraron capacidades similares para la difusión de los túbulos dentinarios. No es necesario un vehículo de baja tensión para transportar los medicamentos a través de los conductos radiculares (Sungur et al.). 
Fuss et al. demostraron que la adición de yodo yoduro de potasio o cobre activado eléctricamente al hidróxido de calcio aumentó significativamente su actividad antibacteriana y su difusión en los túbulos dentinarios in vitro. Recomendando que se necesitan más estudios para identificar agentes antibacterianos no tóxicos que sean más efectivos y sistemas de administración que aseguren el contacto directo del medicamento con las bacterias en los túbulos dentinarios (Fuss et al.).

Con respecto al diámetro de las partículas de la medicación intraconductos, Sireesha et al. obtuvieron como conclusión de su estudio in vitro que la medicación intraconductos de hidróxido de calcio y chitosan elaborada con nanopartículas demostraron una penetración superior dentro del túbulo dentinal con un apreciable efecto antibacteriano, cuando se comparó con los mismos medicamentos, pero con partículas de diametros mayores (Sireesha et al.).

Gravenmade et al. (1981), concluyeron que en personas jóvenes (menores de 20 años), toda la superficie radicular es permeable, posiblemente porque los túbulos dentinales en pacientes jóvenes poseen una calcificación peritubular mínima que es capaz de absorber gran parte del medicamento en el conducto, mientras que en dientes de personas de mayor edad, la hipercalcificación en la dentina periférica de la raiz es otra razón para explicar la impermeabilidad de los medicamentos (Gravenmade et al.).

Con respecto a la difusión del medicamento en las complejidades anatómicas del diente, Gravenmade et al., indican que la mayor parte del formaldehído insertado se difundió a través del tercio apical. Esto puede explicarse porque la difusión tiene lugar principalmente a través de los conductos laterales y sus ramificaciones.

Un concepto interesante con respecto a la efectividad del medicamento dentro del conducto radicular, es la zona de inhibición, lo cual indicaría la actividad contra los microorganismos, su efectividad depende de varios factores, como el tamaño de la molécula, solubilidad y difusión del material en el medio de agar, sensibilidad a la droga, origen bacteriano, número de bacterias inoculadas, $\mathrm{pH}$ del sustrato, viscosidad del agar, tiempo de incubación y actividad metabólica en contra del microorganismo (Silva et al., 2014). Motivo por el cual, en el presente trabajo no se evalua la "zona de inhibición", por ser un concepto tan amplio.

\section{CONCLUSIONES}

No se han reportado estudios suficientes en la literatura que reporten la capacidad de penetración y difusión de la medicación intraconductos dentro del túbulo dentinal, conductos laterales e itsmos. La eliminación de la capa de desecho aumentaría la capacidad de penetración/difusión del medicamento dentro del túbulo dentinal. El vehículo empleado en la preparación del medicamento intraconductos y el diámetro de las partículas del mismo, incide en la capacidad de penetración/difusión del medicamento dentro del túbulo dentinal, conductos laterales e itsmo. Se determinó un rango de penetración del medicamento muy variable, entre $27,7-2350 \mu \mathrm{m}$ y rango de difusión de 200-500 $\mu \mathrm{m}$.

JIMÉNEZ, R. L. F.; JUAREZ, M. P. \& FERREIRA, A. F. R. Penetration and diffusion capacity of intra-duct medication in dentinal tubules, lateral canals and isthmus. A systematic review. Int. J. Odontostomat., 15(3):727-733, 2021.

ABSTRACT: The objective of endodontic therapy is to treat or prevent apical periodontitis. The effective elimination or reduction of the microbiota is essential for periapical repair. Due to this, the use of intracanal drugs is used to chemically treat the root canal system, and the penetration and diffusion capacity of said drug becomes relevant to come into direct contact with the microorganisms that penetrate inside the dentinal tubules, lateral canals and isthmus. A literature review was carried out in electronic databases with the search engines "Pubmed, Scielo and Google Academic", using keywords to identify the relevant published literature in English. The analysis and eligibility criteria were documented according to the reporting items for systematic reviews and meta-analyzes (Preferred Reporting Items for Systematic Reviews and Meta-Analysis, PRISMA). No restrictions were placed on the date of publication. Data on intracanal drug diffusion capacity in dentinal tubules, lateral canals and root isthmus were collected and subjected to descriptive data analysis. The bibliographic search in electronic databases according to the inclusion criteria yielded 45 titles and abstracts. Among them, 7 articles were selected. A highly variable drug penetration range was determined, between 27.7 $-2350 \mu \mathrm{m}$ and a diffusion range of $200-500 \mu \mathrm{m}$. There are few published studies that report the penetration and diffusion capacity of intracanal medication. The vehicle used and the diameter of the particles in the preparation of the drug and the elimination of the waste layer would increase the penetration / diffusion capacity of the drug within the dentinal tubule, lateral canals and isthmus.

KEY WORDS: Lateral canal, root isthmus, intracanal medication, penetration, diffusion, dentinal tubule. 


\section{REFERENCIAS BIBLIOGRÁFICAS}

Abbott, P. \& Yu, C. A clinical classification of the status of the pulp and the root canal system. Aust. Dent J., 52(1 Suppl.):S17-S31, 2007.

Abbott, P. V.; Heithersay, G. S. \& Hume, W. R. Release and diffusion through human tooth roots in vitro of corticosteroid and tetracycline trace molecules from Ledermix paste. Endod. Dent. Traumatol., 4(2):55-62, 1988

Abbott, P. V.; Hume, W. R. \& Heithersay, G. S. Barriers to diffusion of Ledermix paste in radicular dentine. Endod. Dent. Traumatol., 5(2):98104, 1989.

Al-Nazhan, S.; Al-Salaiman, A.; Al-Rasheed, F.; Alnajiar, F.; Al-Abdulwahab, B. \& Al-Badah, A. Microorganism penetration in dentinal tubules of instrumented and retreated root canal walls. In vitro SEM study. Restor. Dent. Endod., 39(4):258-64, 2014.

American Association of Endodontists. Root Canal Irrigants and Disinfectans, 2011.

Arathi, G.; Rajakumaran, A.; Divya, S.; Malathi, N.; Saranya, V. \& Kandaswamy, D. Comparison of penetrating depth of chlorhexidine and chitosan into dentinal tubules with and without the effect of ultrasonic irrigation. J. Oral Maxillofac. Pathol., 23 (3):389-32, 2019.

Brittan, J. L.; Sprague, S. V.; Macdonald, E. L.; Love, R. M.; Jenkinson, H. F. \& West, N. X. In vivo model for microbial invasion of tooth root dentinal tubules. J. Appl. Oral Sci., 24(2):126-35, 2016.

Byström, A. \& Sundqvist, G. Bacteriologic evaluation of the efficacy of mechanical root canal instrumentation in endodontic therapy. Scand. J. Dent. Res., 89(4):321-8, 1981.

Cruz, E. V.; Kota, K.; Huque, J.; Iwaku, M. \& Hoshino, E. Penetration of propylene glycol into dentine. Int. Endod. J., 35(4):330-6, 2002.

de Souza Batista, V. E.; Olian, D. D. \& Mori, G. G. Diffusion of Hydroxyl ions from calcium hydroxide and aloe vera pastes. Braz. Dent. J., 25(3):212-6, 2014.

El Karim, I.; Kennedy, J. \& Hussey, D. The antimicrobial effects of root canal irrigation and medication. Oral Surg. Oral Med. Oral Pathol. Oral Radiol. Endod., 103(4):560-9, 2007.

Estrela, C.; Rodrigues de Araújo Estrela, C.; Bammann, L. L. \& Pecora, J. D. Two methods to evaluate the antimicrobial action of calcium hydroxide paste. J. Endod., 27(12):720-3, 2001.

Fuss, Z.; Mizrahi, A.; Lin, S.; Cherniak, O. \& Weiss, E. I. A laboratory study of the effect of calcium hydroxide mixed with iodine or electrophoretically activated copper on bacterial viability in dentinal tubules. Int. Endod. J., 35(6):522-6, 2002.

Gravenmade, E. S.; Wemes, J. \& Dankert, J. Quantitative measurements of the diffusion in vitro of some aldehydes in root canals of human teeth. Oral Surg., 52(1):97-100, 1981.

Haapasalo, M. \& Orstavik, D. In vitro infection and disinfection of dentinal tubules. J. Dent. Res., 66(8):1375-9, 1987.

Lasala, A. Guía de Endodoncia Clínica. Maracaibo, Venezuela, Editorial Universitaria L.U.Z., 1971.

Law, A. \& Messer, H. An evidence-based analysis of the antibacterial effectiveness of intracanal medicaments. J Endod., 30(10):689-94, 2004.

Liberati, A.; Altman, D. G.; Tetzlaff, J.; Mulrow, C.; Gøtzsche, P. C.; loannidis, J. P. A.; Clarke, M.; Devereaux, P. J.; Kleijnen, J. \& Moher, D. The PRISMA statement for reporting systematic reviews and meta-analyses of studies that evaluate healthcare interventions: explanation and elaboration. PLoS Med., 339:b2700, 2009.

Lin, S.; Levin, L.; Peled, M.; Weiss, E. I. \& Fuss, Z. Reduction of viable bacteria in dentinal tubules treated with clindamycin or tetracycline. Oral Surg. Oral Med. Oral Pathol. Oral Radiol. Endod., 96(6):751-6, 2003.

Mohammadi, Z. \& Abbott, P.V. Antimicrobial substantivity of root canal irrigants and medicaments: a review. Aust. Endod. J., 35(3):131-9, 2009.

Oguntebi, B. R. Dentine tubule infection and endodontic therapy implications. Int. Endod. J., 27(4):218-22, 1994.

Ørstavik, D. \& Haapasalo, M. Disinfection by endodontic irrigants and dressings of experimentally infected dentinal tubules. Endod. Dent. Traumatol., 6(4):142-9, 1990.
Peciuliene, V.; Reynaud, A. H.; Balciuniene, I. \& Haapasalo, M. Isolation of yeasts and enteric bacteria in root-filled teeth with chronic apical periodontitis. Int. Endod. J., 34(6):429-34, 2001.

Peters, L. B.; Wesselink, P. R. \& Moorer, W. R. Penetration of bacteria in bovine root dentine in vitro. Int. Endod. J., 33(1):28-36, 2000.

Portenier, I.; Haapasalo, H.; Orstavik, D.; Yamauchi, M. \& Haapasalo, M. Inactivation of the antibacterial activity of iodine potassium iodide and chlorhexidine digluconate against Enterococcus faecalis by dentin, dentin matrix, type-I collagen, and heat-killed microbial whole cells. $J$. Endod., 28(9):634-7, 2002

Puapichartdumrong, P.; Ikeda, H. \& Suda, H. Influence of the pulpal components on human dentine permeability in vitro. Int. Endod. J., 38(3):152-9, 2005.

Ran, S.; Wang, J.; Jiang, W.; Zhu, C. \& Liang, J. Assessment of dentinal tubule invasion capacity of Enterococcus faecalis under stress conditions ex vivo. Int. Endod. J., 48(4):362-72, 2015.

Seltzer, S. Pain in endodotics. J. Endod., 30(7):501-3, 2004.

Sen, B.; Piskin, B. \& Demirci, T. Observation of bacteria and fungi in infected root canals and dentinal tubules by SEM. Endod. Dent. Traumatol., 11:6-9, 1995.

Silva, A.; Brasil, E.; Souza, S.; Gomes, J.; Vaz, I. \& Fontes, M. Antimicrobial effect and transdentinal diffusion of new intracanal formulations containing nitrofurantoinord oxycycline. Braz. Dent. J., 25(5):425-9, 2014

Siqueira Jr. J. \& Lopes, H. Mechanisms of antimicrobial activity of calcium hydroxide: a critical review. Int. Endod. J., 32:361-9, 1999.

Siqueira Jr., J. F.; De Uzeda, M. \& Fonseca, M. E. A scanning electron microscopic evaluation of in vitro dentinal tubules penetration by selected anaerobic bacteria. J. Endod., 22(6):308-10, 1996.

Siqueira Jr., J.; Machado, A.; Silveira, R.; Lopes, H. \& De Uzeda, M. Evaluation of the effectiveness of sodium hypochlorite used with three irrigation methods in the elimination of enterococcus faecalis from the root canal, in vitro. Int. Endod. J., 30:279-82, 1997.

Siqueira Jr. J.; Rôças, I.; Magalhães, F. \& Uzeda, M. Antifungal effects of endodontic medicaments. Aust. Endod. J., 27(3):112-4, 2001

Sireesha, A.; Jayasree, R.; Vidhya, S.; Mahalaxmi, S.; Sujatha, V. \& Sampath, T. Comparative evaluation of micron- and nano-sized intracanal medicaments on penetration and fracture resistance of root dentin - An in vitro study. Int. J. Biol. Macromol., 104(Pt. B):1866-73, 2017.

Sjögren, U.; Figdor, D.; Spangberg, L. \& Sundqvist, G. The antimicrobial effect of calcium hydroxide as a short-term intracanal dressing. Int. Endod. J., 24:119-25, 1991.

Sungur, D.; Aksel, H. \& Purali, N. Effect of a low Surface tension vehicle on the dentinal tubule penetration of calcium hydroxide and triple antibiotic paste. J. Endod., 43(3):552-5, 2017.

Wantulok, J.; Wash, S. \& Brown, J. An in vitro study of the diffusibility of camphorated parachlorophenol and metacresylacetate in the root canal. Oral Surg., 34(4):653-60, 1972.

Wu, M.; Dummer, P. \& Wesselink, P. Consequences of and strategies to deal with residual post-treatment root canal infection. Int. Endod. J., 39:343-56, 2006.

Zand, V.; Mokhtari, H.; Hasani, A. \& Jabbari, G. Comparison of the penetration depth of convencional and nano-particle calcium hydroxide into dentinal tubules. Iran. Endod. J., 12(3):366-70, 2017.

Zou, L.; Shen, Y.; Li, W. \& Haapasalo, M. Penetration of sodium hypochlorite into dentin. J. Endod., 36:793-6, 2010.

Dirección para correspondencia:

Luis Felipe Jiménez Rojas

Ciudad Universitaria de Caracas

Los Chaguaramos Caracas

Código Postal: 1050. Rif: G-20000062-7.

VENEZUELA

E-mail: luisfelipejimenezrojas@gmail.com luisfelipe_jimenez@hotmail.com 